\title{
Erratum to: Mixed Broadcast Techniques of Leisure Information in Vehicular Ad-Hoc Networks
}

\author{
Pei-Wei Tsai ${ }^{2} \cdot$ Jian-Pan Li $^{1} \cdot$ Jia-Shing Shih $^{3} \cdot$ Yin-Jun Chen $^{3} \cdot$ Tung-ying Lee ${ }^{4}$. \\ Sheng-Tzong Cheng ${ }^{3}$
}

Published online: 19 April 2016

(c) Springer Science+Business Media New York 2016

\section{Erratum to: Telecommun Syst \\ DOI 10.1007/s11235-016-0158-2}

The listing of the author affiliations in the published article is incorrect. Instead of just one affiliation, there should be four affiliations as listed in this erratum.

The online version of the original article can be found under doi: 10.1007/s11235-016-0158-2.

Jian-Pan Li

keyboardicrd@gmail.com

1 National Chung-Shan Institute of Science \& Technology,

Taoyuan, Taiwan

2 Fujian University of Technology, Fujian, China

3 National Cheng-Kung University, Tainan, Taiwan

4 National University of Tainan, Tainan, Taiwan 